\title{
Particle Dynamics Modelling of Cell Populations
}

\author{
N. Bessonov ${ }^{1,2}$, P. Kurbatova ${ }^{2}$, V. Volpert ${ }^{2 *}$ \\ ${ }^{1}$ Institute of Mechanical Engineering Problems, 199178 Saint Petersburg, Russia \\ ${ }^{2}$ Institute Camille Jordan, University Lyon 1, UMR 5208 CNRS, 69622 Villeurbanne, France
}

\begin{abstract}
Evolution of cell populations can be described with dissipative particle dynamics, where each cell moves according to the balance of forces acting on it, or with partial differential equations, where cell population is considered as a continuous medium. We compare these two approaches for some model examples.
\end{abstract}

Key words: particles dynamics, continuous medium, point source AMS subject classification: 70F40, 35J05

\section{Particle dynamics and continuum mechanics}

Dissipative particles dynamics is developed as a method of simulation of complex media [1]. We apply it in order to study biological cell populations taking into account possible cell division, differentiation and death [2]. In this note we carry out a more detailed comparison between particle dynamics and the corresponding equations of continuum mechanics.

\subsection{Particles and discrete equations}

Consider a system of $N$ particles in the plane. Denote their coordinates by $x_{1}, \ldots, x_{n}$. Here $x_{i}$ is a two-component vector. Suppose that all particles have the same mass $m$ and consider the equation of motion of the $i$-th particles in the form

$$
m \ddot{x}_{i}+\mu m \dot{x}_{i}-\sum_{j \neq i} f_{i j}=0 .
$$

\footnotetext{
${ }^{*}$ Corresponding author. E-mail: volpert@ math.univ-lyon1.fr
} 
The dot denotes the derivative with respect to time, $\ddot{x}_{i}$ is the particle acceleration, $\dot{x}_{i}$ is its speed. The second term in the left-hand side of this equation describes dissipation due to friction, the last term is the sum of forces acting on this particle from all other particles. The force $f_{i j}$ acting between the particles $i$ and $j$ can be expressed through the potential: $f_{i j}=-m \nabla \phi\left(\left|x-x_{j}\right|\right)_{x=x_{i}}$. Then

$$
\ddot{x}_{i}+\mu \dot{x}_{i}+\nabla\left(\sum_{j \neq i} \phi\left(\left|x-x_{j}\right|\right)\right)_{x=x_{i}}=0, \quad 1, \ldots, N
$$

Consider a square grid with the mesh points $\bar{x}_{i}$ and the step $\delta x$. Denote by $s_{i}$ the square with the side $2 \delta x$ and the center at the point $\bar{x}_{i}$. Let $x_{i 1}, \ldots, x_{i k} \in s_{i}$. Let us introduce velocity and density at the grid points:

$$
v_{i}=\frac{1}{k} \sum_{j=1}^{k} \dot{x}_{i j}, \quad \rho_{i}=\frac{k}{\left|s_{i}\right|}
$$

where $\left|s_{i}\right|=4(\delta x)^{2}$ is the area of $s_{i}$. Then

$$
\sum_{j} \phi\left(\left|x-x_{j}\right|\right) \approx \sum_{m} \phi\left(\left|x-\bar{x}_{m}\right|\right) \rho_{m}\left|s_{m}\right| \approx U(x)
$$

where

$$
U(x)=\int \phi\left(\left|x-x^{\prime}\right|\right) \rho\left(x^{\prime}\right) d x^{\prime} .
$$

Taking a sum of equations (1.2) with respect to the points inside $s_{i}$, we obtain the discrete equation

$$
\rho_{i} \frac{d v_{i}}{d t}+\mu \rho_{i} v_{i}+\rho_{i}(\nabla U)_{i}=0
$$

where we use the approximation $\frac{1}{k} \sum_{j=1}^{k} \phi\left(\left|x_{j}-\bar{x}_{m}\right|\right) \approx \phi\left(\left|\bar{x}_{i}-\bar{x}_{m}\right|\right)$. Averaged equation (1.3) may not be equivalent to (1.2). Two particles $x_{j}, x_{k} \in s_{i}$ with opposite velocities cancel in (1.3) but not in (1.2). They result in the momentum transfer and can be taken into account in (1.3) by additional dissipative terms. In numerical simulations, the equivalence of these equations can be provided if we take an average velocity with respect to some ensembles of particles.

\subsection{Continuous model}

Consider the continuous analogue of discrete equation (1.3)

$$
\rho \frac{d v}{d t}+\mu \rho v+\rho \nabla U=0
$$

This equation of motion should be completed by the equation of mass conservation:

$$
\frac{\partial \rho}{\partial t}+\nabla \cdot(\rho v)=0
$$


We can write equation (1.4) in the form

$$
\rho\left(\frac{\partial v}{\partial t}+v \cdot \nabla v\right)+\mu \rho v+\nabla p-U \nabla \rho=0,
$$

where $p$ is the pressure, $p=\rho U$. This equation is nonlocal because the potential $U$ contains the integral. Using the Taylor expansion of the density $\rho\left(x^{\prime}\right)$ around the point $x$ and keeping the terms up to the second order, we get rid of the integral and obtain the equation

$$
\rho\left(\frac{\partial v}{\partial t}+v \cdot \nabla v\right)+\mu \rho v+\nabla p-K \Delta \rho \nabla \rho=0 .
$$

The pressure in this equation is different in comparison with the previous one but we keep for it the same notation. This equation does not contain the potential anymore. Therefore, we cannot express the pressure through it, and we need to complete system (1.5), (1.7) by an equation of state, $p=p(\rho)$. In the case $\mu=0$ and $K=0,(1.7)$ is the Euler equation and system (1.5), (1.7) becomes the classical model of gas dynamics.

\subsection{Energy}

Denote the left hand-side of equation (1.4) by $J(x, t)$. We take a scalar product of this vector with $v$ and integrate over $\mathbb{R}^{2}$ :

$$
\begin{gathered}
\int J v d x=\frac{1}{2} \int \rho \frac{\partial|v|^{2}}{\partial t} d x+\frac{1}{2} \int\left(\rho v, \nabla|v|^{2}\right) d x+\mu \int \rho|v|^{2} d x+\int(\rho v, \nabla U) d x= \\
\frac{1}{2} \frac{d}{d t} \int\left(\rho|v|^{2}+\rho U\right) d x+\mu \int \rho|v|^{2} d x .
\end{gathered}
$$

We use here formal integration by parts, equation (1.5) and the equality

$$
\int \frac{\partial \rho}{\partial t} U d x=\frac{1}{2} \frac{d}{d t} \iint \phi\left(\left|x-x^{\prime}\right|\right) \rho(x, t) \rho\left(x^{\prime}, t\right) d x d x^{\prime}=\frac{1}{2} \frac{d}{d t} \int \rho U d x .
$$

From (1.4) we obtain

$$
\frac{d E}{d t}=-\mu \int \rho|v|^{2} d x
$$

where $E$ is the sum of the kinetic and potential energy of the system:

$$
E=\frac{1}{2} \int \rho|v|^{2} d x+\frac{1}{2} \int \rho U d x
$$

It follows from (1.8) that the total energy of the system decreases, its kinetic energy tends to zero while the potential energy to some constant. Total energy can also be introduced for the system of particles. Similar to the continuous case, it decreases with time. 


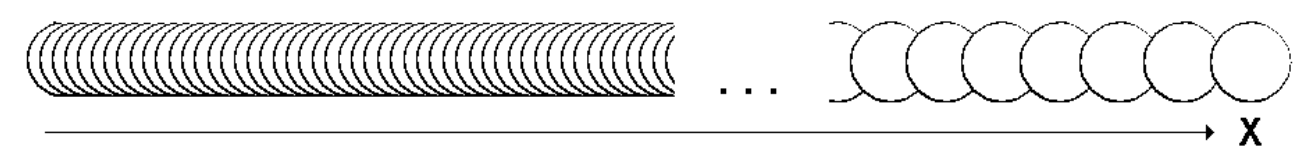

Figure 1: Particle flow in 1D model problem.
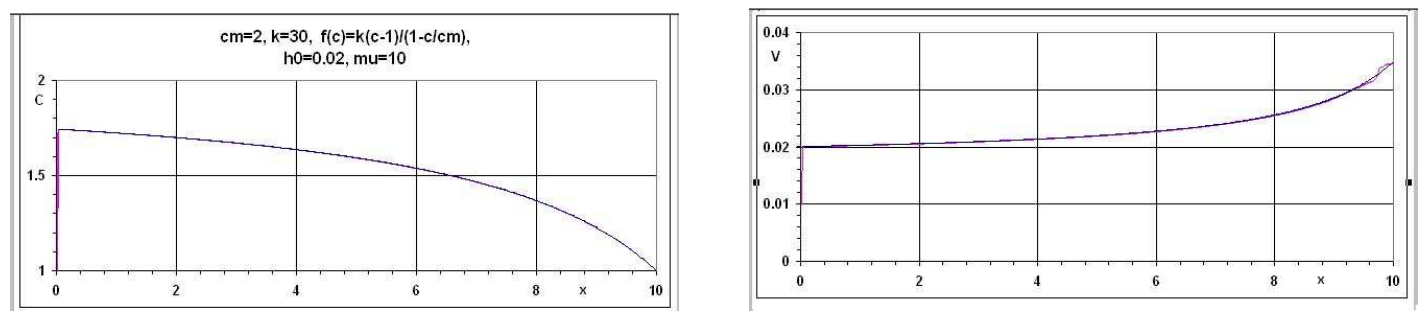

Figure 2: Particle dynamics (red) and analytical solution (black) for 1D particle flow, concentration (left) and velocity (right) of particles.

\section{Model examples}

\subsection{D case}

In the 1D case, the last term in the left-hand side of the equation (1.7) has the form $K \rho^{\prime} \rho^{\prime \prime}$ and can be included in the pressure. In the stationary case, neglecting the inertial terms, we obtain from (1.5), (1.7):

$$
\mu \rho v+p^{\prime}=0, \quad \rho v=q,
$$

where $q$ is some constant. The force between two spherical particles is considered in the form

$$
f(h)=\left\{\begin{array}{ccc}
k \frac{h_{0} / h-1}{2-h_{0} / h} & , & h_{0} / 2<h<h_{0}, \\
0 & , & h>h_{0}
\end{array}\right.
$$

where $h$ is the distance between their centers, $k$ and $h_{0}$ are positive parameters. The force between the particles tends to infinity when $h$ decreases to $h_{0} / 2$. On the other hand, this force equals zero if $h \geq h_{0}$. Hence, every given particles interacts with at most one other particle from the left of it and another one from the right.

If $c=h_{0} / h$ is the number of particles in the interval of the length $h_{0}, c=h_{0} \rho$, then we obtain from the previous formula the equation of state, $p(c)=k(c-1) /(2-c)$. Together with (2.1) it allows us to find the function $c(x)$. Figure 1 shows an example of particle dynamics simulations, and Figure 2 the comparison of numerical simulations of particle dynamics with the analytical solution. The numerical and analytical curves exactly coincide. 

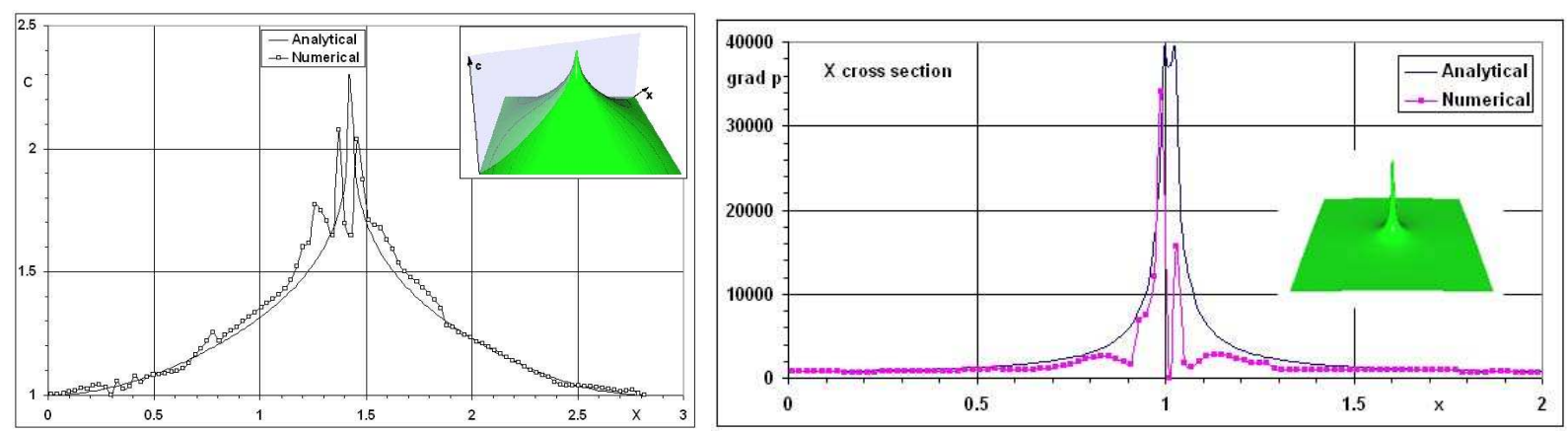

Figure 3: Left: concentration distribution for the discrete (dotted line) and continuous (smooth line) models along the diagonal of the square computational domain (upper right corner). Right: the modulus of $\nabla p$ along the $x$-direction.

\subsection{D case}

In the 2D model example we consider a square domain with a fixed dividing particle in its center. This particle produces other spherical particles with a given frequency. The direction of division, that is the angle of newly born particles with respect to the original particle is random. Thus we have a point source of particles from the center of the domain. The corresponding continuous model in the stationary case can be derived from equations (1.4), (1.5):

$$
\Delta p=J(x), x \in \Omega, \quad p=0, x \in \partial \Omega .
$$

Here $J(x)$ is a Dirac $\delta$-function, in the case of a point source, or its approximation.

This problem allows us to determine the pressure distribution. We need an equation of state to find the concentration. If the particles are locally uniformly distributed and form a square grid, then $c=\left(h_{0} / h\right)^{2}$. Simple computations allow us to find the expression for the pressure from the expression for the force between two neighboring particles (2.2):

$$
p(c)=k \sqrt{c} \frac{\sqrt{c}-1}{2-\sqrt{c}} .
$$

A similar formula, though quantitatively different, holds if the packing of particles is different. In particular, it can be hexagonal.

Thus, we can determine the concentration distribution from particle dynamics and from the equations of continuum mechanics. Their comparison is shown in Figure 3.

\section{Motion of dividing cells}

Biological cells can divide and create a cell population around them. Appearance of new cells creates cell pressure and, as a consequence, their motion. This motion changes the position of the dividing cells. Thus, we come to the problem about the motion of dividing cells. From the biological point of view this is related to the distribution of stem cells, which possess the capacity 

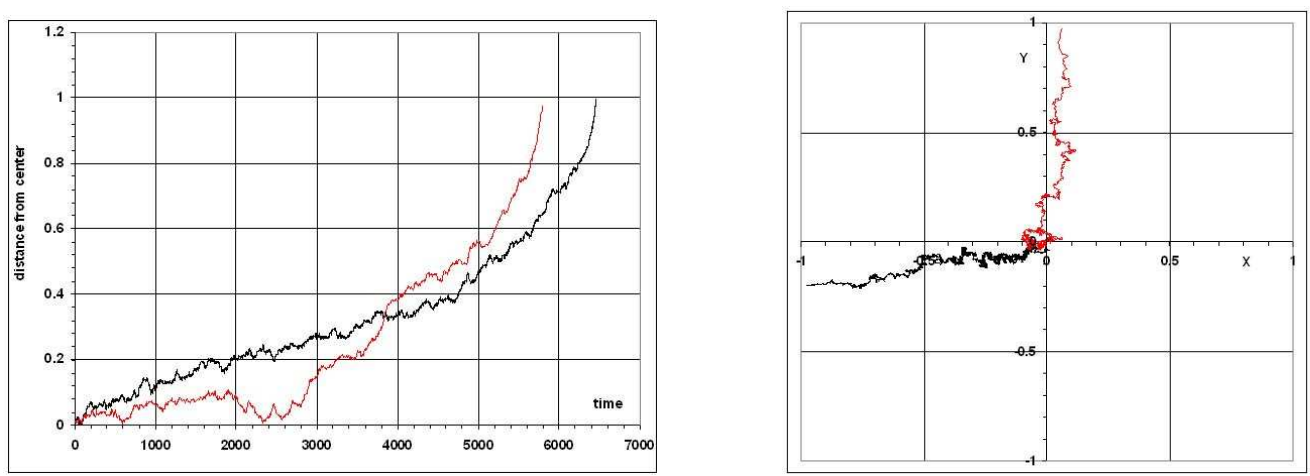

Figure 4: Motion of a dividing particle, distance from the center of the domain (left) and its trajectory (right). Two curves correspond to two different simulations.

to self-renew. Figure 4 shows the motion of a single dividing cell in a square filled by non-dividing cells. The dividing cell feels the shortest way to the wall and moves in this direction.

The corresponding continuous problem writes

$$
\Delta p=\delta(x-\xi(t)), x \in \Omega, \quad p=0, x \in \partial \Omega, \quad \xi^{\prime}(t)=\oint p d s,
$$

where $\xi(t)$ is a position of the point source. It is a $2 \mathrm{D}$ vector. The integral is taken over a contour around the point $\xi$. Solution of this problem can be sought as $p(x, t)=p_{0}(x, t)+a \ln (b / \mid x-$ $\xi(t) \mid)$, where $p_{0}$ is a regular harmonic function with non-homogeneous boundary conditions. Then $\xi^{\prime}(t)=\nabla p_{0}(\xi(t), t)$. Numerical solution of this problem shows the same qualitative behavior as for particle dynamics. The point source moves to the boundary by the shortest way. Its speed increases when it approaches the wall.

\section{References}

[1] M. Karttunen, I. Vattulainen, A. Lukkarinen. A novel methods in soft matter simulations. Springer, Berlin, 2004.

[2] N. Bessonov, L. Pujo-Menjouet, V, Volpert. Cell modelling of hematopoiesis. Math. Model. Nat. Phenom., 1 (2006), No. 2, 81-103. 